\title{
Exact diagonalization Studies of Two-dimensional Frustrated Antiferromagnet Models
}

\author{
C. Lhuillier, P. Sindzingre, J.-B. Fouet \\ Laboratoire de Physique Théorique des Liquides-UMR 7600 of CNRS, Université Pierre et Marie Curie, case 121, 4 place \\ Jussieu, 75252 Paris Cedex, France \\ E-mail:lhuilier@lptl.jussieu.fr
}

(November 2, 2018)

PACS numbers: 75.10.Jm; 75.50.Ee; 75.40.-s

We describe the four kinds of behavior found in twodimensional isotropic quantum antiferromagnets. Two of them display long range order at $T=0$ : the Néel state and the Valence Bond Crystal. The last two are Spin-Liquids. Properties of these different states are shortly described and open questions are underlined.

\section{INTRODUCTION}

Exact diagonalizations are restricted to small spin systems (up to about $N=36$ spins with the present day computers). Some problems as the study of critical regimes are out of reach of such an approach, on the contrary we think that this approach might help in answering other subtle questions as for example symmetry breaking, order out of disorder, qualitative nature of the groundstate and of the first excitations of two-dimensional (2d) spin- $1 / 2$ magnets.

We have used it extensively to study the competition of long range order (LRO) versus "quantum disorder" at $T=0$. Up to now, by looking to the spectra of model spin systems, we have been able to characterize four kinds of behavior: Néel LRO (collinear or not), dimer LRO and two different Spin-Liquids. In this paper we will give a brief picture of these four generic cases of $2 \mathrm{~d}$ magnetism, underline the more efficient numerical criteria to determine the nature of a given unknown system and try to extract from the present numerical knowledge what are the conditions for the appearance of the four different phases. The key point of our approach is the analysis of the symmetries and finite size scaling of the low lying eigen-levels of a given hamiltonian on small samples with periodic (or eventually twisted) boundary conditions. The evolution of the spectrum with the size and shapes of the sample has to be thoroughly understood before conclusion: it may be a lengthy and sometimes subtle process but it is usually quite valuable. Some examples will be quoted in the following sections.

\section{NÉEL LONG RANGE ORDER}

There is no mathematical proof that Néel long range order exists at $T=0$ in $2 \mathrm{~d}$ spin- $1 / 2$ systems. But there is a very large consensus that it should be the case for the Heisenberg nearest neighbor hamiltonian on bipartite lattices [1]. Exact diagonalizations and/or Monte-Carlo calculations were performed for the Heisenberg model on the square lattice [2], on the triangular lattice [3, 1] and on the honeycomb lattice [5]. On each of these lattices the Heisenberg model displays long range order with a sublattice magnetization reduced to $60 \%$ of the classical value on the square lattice $(\sim 40 \%$ on the triangular lattice [3, 1 , $44 \%$ on the honeycomb lattice [5],6]). These values of the order parameter might be in error by a few percent: in these antiferromagnets with linear Goldstone modes, the scaling law for the magnetization of samples of linear size $L \sim N^{1 / d}$ is an expansion in $1 / L$ (i.e. $1 / N^{1 / 2}$ in 2 d) [7, 8]. For the sizes encountered in exact diagonalizations, the asymptotic $1 / N^{1 / 2}$ law is never reached [9, 10] and the extrapolation to the thermodynamic limit remains uncertain: nevertheless the agreement between the scaling behaviors of diagonalization results and spin wave results gives a strong support to the above-mentioned conclusions.

A stronger confirmation of Néel LRO is obtained by the scaling of the spectrum itself, which is an illuminating illustration of the mechanism of symmetry breaking. In order to understand in a simple way how a continuous symmetry breaking manifests itself on finite size spectra, let us consider a piece of solid. Our experience of the macroscopic solid is the following: it breaks the translation symmetry and its center of mass (c.o.m.) is localized in space. On the other hand, we can sort its degrees of freedom in two parts: first the c.o.m. coordinates and the internal variables. The hamiltonian of such a problem breaks in two commuting parts: the c.o.m. kinetic energy and the hamiltonian of the internal degrees of freedom ( in a solid, in a first approximation this second part is a phonon hamiltonian). For a finite size sample of $\mathrm{N}$ atoms, with mass $\mathrm{m}$, this reads:

$$
H_{t o t}=\frac{\mathbf{P}^{2}}{2 m N}+h_{\text {phonons }} .
$$

The total hamiltonian does not break translation symmetry, the total momentum $\mathbf{P}$ is a constant of the motion and its eigenvalues good quantum numbers. Due to the commutation property of the sub-parts of the hamiltonian, the eigenstates are direct products of eigenstates of the c.o.m. kinetic energy and phonons eigenstates. From these very simple observations it follows that the spectrum of Eq. 1 as a function of the eigenvalues of the 
square of the momentum has the appearance described in Fig. 1. A straight line is drawn across the eigen-energies of the c.o.m. hamiltonian with 0 phonon (respectively 1 phonon...). Let us first concentrate on the vacuum of phonons: the associated eigen-levels collapse to the absolute ground-state as $1 / N$ when $\mathrm{N}$ goes to infinity. Let us now consider the eigenlevels immediately above this family: they correspond to the softest phonon excitation and are distant from the vacuum family by the energy of this phonon, that is an energy $\mathcal{O}(1 / L) \rrbracket$. When the size goes to infinity, if the dimension of the solid is equal or larger than two, the levels forming the vacuum of phonons collapse to the absolute ground-state faster than the softest phonon! This allows to form a wave packet out of these levels and localize the c.o.m. with a cost in energy lower than the softest phonon: the solid phase with its specific macroscopic properties (symmetry breaking and stiffness) can exist in $2 \mathrm{~d}$ (at $T=0$ ), whereas it cannot exist quantum mechanically in $1 \mathrm{~d}$.

Let us now turn to the Néel ordered magnet. For simplicity we will first consider a collinear magnet: the macroscopic state of the magnet at $T=0$ is defined by the Euler angles of the order parameter. They are the equivalent of the c.o.m. coordinates in the solid problem. The conjugate variables of these Euler angles are the components of the total spin and their free precession is described by the Hamiltonian:

$$
H_{\text {coll.var. }}=\frac{\mathbf{S}^{2}}{2 \chi N}
$$

This is the equation of motion of a rigid rotor. The moment of inertia of this rotor is written here in terms of the intensive homogeneous spin susceptibility $\chi, N$ is the number of spins. If the system has LRO and rigidity, the motion of the internal variables is governed by an hamiltonian of (antiferromagnetic ) magnons and the total hamiltonian reads:

$$
H_{\text {tot }}=\frac{\mathbf{S}^{2}}{2 \chi N}+h_{\text {magnons }} .
$$

When $H_{t o t}$ is $S U(2)$ invariant, $\mathbf{S}^{2}$ is a constant of motion and its eigenvalues $S(S+1)$ are good quantum numbers. It is thus interesting to display the spectrum of this ordered antiferromagnet as a function of $S(S+1)$. Its generic appearance is shown in Fig. 2. One recognizes the (Pisa-)tower of states associated with the vacuum of magnons $|0\rangle$ (these states are called QDJS for Quasi Degenerate Joint States in refs. [3,90, and the tower of states associated with the softest magnon $|1\rangle$. The semi-classical Néel ground-state with an order parameter pointing in the $(\theta, \phi)$ direction is a wave packet

\footnotetext{
${ }^{1}$ This energy has to be measured along the arrow in Fig 1: this takes care of the momentum of the phonon.
}

of eigen-levels with different total spin belonging to the QDJS $|0\rangle$. Mixing different $\mathrm{S}$ values up to $\sqrt{N}$ allows the localization of the order parameter. If the levels of the $|0\rangle$ family collapse to the ground-sate faster than the softest magnon, the classical picture survives to quantum fluctuations and the system exhibits LRO: as for the solid, that might be the case at $T=0$ for $2 \mathrm{~d}$ magnets. For $T \neq 0$, thermal fluctuations destroy the order (Mermin-Wagner theorem). Notice that the entropy of the semi-classical ground-state scales as $\operatorname{Ln}\left(N^{2}\right)$, and the entropy per spin of the ordered ground-state is zero in the thermodynamic limit.

The levels associated to the vacuum of magnons have special properties with respect to the lattice symmetry group: these properties reveal the space symmetry breaking of the Néel state. Let us take as an example the collinear order on the honeycomb lattice (Fig. 3): it does not break the translational symmetry of the Bravais lattice (thus only the $\mathbf{k}=\mathbf{0}$ momentum appears in the QDJS). But it breaks both the inversion symmetry with respect to the center of an hexagon and the reflexion with respect to an axis going through nearest neighbor hexagon centers, and it is invariant in the product of these two symmetries. Thus both the even and odd irreducible representations of these two symmetry groups appear in the QDJS (symbols $R_{\pi}= \pm 1, \sigma= \pm 1$ ), but they are always associated so that

$$
R_{\pi} \cdot \sigma=+1
$$

which insures the invariance in the product of the two symmetries, and

$$
\sigma \cdot(-1)^{S}=+1
$$

which insures the invariance of the semi-classical groundstate in the product of a reflexion times a spin-flip. This is the simplest situation for an antiferromagnet. On the square lattice (which is equally bipartite) Néel order is collinear and Eq. 3 remains valid, but as the translation symmetry is broken, both the $\mathbf{k}=(0,0)$ and $\mathbf{k}=(\pi, \pi)$ vectors appear among the QDJS.

A more complex order parameter implies a richer structure of the QDJS: in the case of the Heisenberg model on the triangular lattice the order parameter is no longer a vector but a trihedron (or equivalently a rotation matrix). Eq.3 is then more complex, involving both the in-plane and out of plane susceptibilities, and there is an internal generation of an extra quantum number (the projection of the total spin on the helicity of the magnet). The free precession hamiltonian is that of a symmetric top 3, 9, 11] and $\mathcal{O}\left(N^{3}\right)$ states collapse to the ground-state in the thermodynamic limit. (Quantum mechanically the QDJS associated to p-sublattice Néel order are adiabatically connected to the states obtained by addition of $p$ spins of size $\frac{N}{2 p}$.) A thorough analysis of the symmetry breaking associated to Néel order on the triangular lattice is done in [9]. 
Unexpectedly the mechanism of "order by disorder" can be detected by looking at the finite size scaling of relatively small samples [12]. We investigated this mechanism in the $J_{1}-J_{2}$ model on the triangular lattice. For $1 / 8<J_{2} / J_{1}<1$ the classical ground-state exhibits a large degeneracy associated to different kinds of 4-sublattice Néel order. For small sizes $(N=16)$ the quantum fluctuations are weak and the vacuum of magnons involve all the levels associated with addition of four momenta. In the $N=28$ sample quantum fluctuations are already strong enough to lift the degeneracy of this family: the levels characteristic of the 2-sublattice Néel order remain the lowest ones in the spectrum, the other ones drift towards the continuum of excitations. It is a nice illustration of the fact that long wave length quantum fluctuations tend to restore the symmetries of the original hamiltonian. The quantum ground-state is more symmetric than the classical one.

Order parameters, spin susceptibilities [9], stiffnesses [10] can be extracted from the numerical data: they are interesting pieces of data to be compared to the spin waves calculations at finite sizes, but as it has been discussed in the beginning of this section, the leading term of their finite size scaling is $\mathcal{O}\left(N^{-\frac{1}{2}}\right)$ and therefore it is more significant to look to the tower of QDJS which collapse as $\mathcal{O}\left(N^{-1}\right)$. It is also simpler to look at the symmetries of the low lying levels and deduce the symmetry of the order parameter than to determine it by direct computations. As a consequence when looking at the disappearance of Néel LRO due to extra frustration or quantum fluctuations it is preferable to pinpoint the range of parameters where the tower of states is destroyed than search for the vanishing of the order parameter [13,14].

\section{DIMER LONG RANGE ORDER AND VALENCE BOND CRYSTALS}

The second class of long-range ordered systems does not imply $S U(2)$ symmetry breaking but only space symmetry breaking. We will call these systems Valence Bond Crystals (VBC) 2: their ground-states are characterized by regular arrays of singlet states on simple bonds or more complicated subsets (4-site plaquettes...). In the thermodynamic limit the ground-state of a VBC is degenerate, with a finite degeneracy directly related to the

\footnotetext{
${ }^{2}$ The name Valence Bond Solid is already "registered" by Affleck, Kennedy Lieb and Tasaki 15, 16 for very specific systems where the individual spins obey the relation $2 S=z$ (with $\mathrm{z}$ the coordination number of the lattice). In such circumstances there exists a whole class of Hamiltonians which have a unique highly symmetric ground-state where each site shares a singlet with any neighbor connected to it.
}

symmetry breaking. The $\mathrm{VBC}$ on the $J_{1}-J_{2}$ chain (for $\frac{J_{2}}{J_{1}}>0.2411 \ldots$ ) is doubly degenerate (it breaks the onestep translation symmetry of the lattice). The simplest VBCs that could be encountered on a triangular lattice have a 12-fold degeneracy (a factor 4 comes from the translation symmetry breaking and a factor 3 from the breaking of $C_{3}$ ). It is a common belief that a VBC has a gap to all excitations (except indeed at critical points).

In $2 \mathrm{~d}$, the more convincing evidence of such a phase, with a spontaneous discrete symmetry breaking has been seen on the $J_{1}-J_{2}$ model on the square lattice (see [2,17] and refs. therein). In that case the VBC breaks translational symmetry but probably not $C_{4}$ : it is probably a long range order of $S=0$ plaquettes.

We have a new example of a VBC in the spectrum of the $J_{1}-J_{2}$ model on the honeycomb lattice. When the second neighbor coupling is strong enough the frustration destroys the collinear Néel long range order and gives birth to a pattern of valence bonds (with a $Z_{3}$ symmetry breaking associated to the three spatial directions for the dimerized bonds). Our predictions is based on the scaling of the low lying singlets of the spectra, it is in agreement with the dimer-dimer correlations functions measured in the first singlet levels supposed to be degenerate in the thermodynamic limit [6].

The nature of the first excitations of these models (coherent or incoherent magnons) remains to be elucidated.

\section{FULLY GAPPED SPIN-LIQUIDS}

Besides these situations with symmetry breaking and LRO in a local observable, we have found two other behaviors, probably generic, with no obvious symmetry breaking, no long range order in a local order parameter. We call these situations "Spin-Liquids" and will successively describe both of them.

Let us first describe very quickly the simplest one which has the following properties (an extensive description of the properties of this phase is given in [18,19]):

- A gap to any excitations

- All spin-spin correlations are short ranged

- A four-fold degeneracy of the ground-state on a 2torus

We have observed this phase for the first time in the multiple-spin exchange problem. This effective hamiltonian has been introduced by Eyring and Thouless to describe the magnetism of quasi localized fermions (electrons in the Wigner Crystal on one hand, ${ }^{3} \mathrm{He}$ atoms in solid phase on the other). The Hamiltonian of the multiple-spin exchange model is given by

$$
H=\sum_{n}(-1)^{n} J_{n}\left(P_{n}+P_{n}^{-1}\right), J_{n}>0, n \geq 2
$$


where $J_{n}$ are the $n$-spin exchange tunneling probabilities (exchange coefficients), $P_{n}$ and $P_{n}^{-1}$ are the $n$-spin permutation operators and their inverse. The alternate sign in the summation over $n$ in Eq. 6 comes from the permutation of fermions. In general, the exchange coefficients decrease with increasing $n$. The two-spin exchange term gives the Heisenberg Hamiltonian up to a constant:

$$
P_{2}=2 \mathbf{S}_{i} \cdot \mathbf{S}_{j}+\frac{1}{2}
$$

where $\mathbf{S}_{i}$ and $\mathbf{S}_{j}$ are spins localized at site $i$ and $j$, respectively. The three-spin exchange operator is equal to a sum of two-spin exchange operators [19]. Thus, the two and three-spin exchange terms are described (up to a constant) by an Heisenberg hamiltonian with an effective coupling constant $J_{2}^{\text {eff }}=J_{2}-2 J_{3}$. Cyclic fourspin exchange gives birth to new physics: it is quartic in spin operators and has built-in frustration. As a result the ground-state of this 4-spin exchange hamiltonian on a single plaquette is doubly degenerate with total spin $S=0$ and 1 ! At the classical level this hamiltonian displays various antiferromagnetic phases 2022$]$, which are wiped out by quantum fluctuations, leading in a large range of parameters to the Spin-Liquid with the abovementioned properties [18, 19].

The four-fold degeneracy of the ground-state on a 2torus is a topological property which was predicted more than ten years ago 23 25. Contrarily to Oshikawa 26] we do not think that it is the signature of long range order in a local order parameter (see discussion in Misguich [19]).

Very few things are clearly stated on the excitations of this Spin-Liquid:

- Plateaus are expected in the magnetization curves both for zero and half the saturation magnetization.

- From the finite size spectra it might be suspected that the triplet excitations form a "continuum" above the spin-gap and that an $S=0$ bound-state exists just below the spin-gap. Up to now, a clear signature of deconfined spinons has not been found in this Spin-Liquid. Futher investigations appear necessary.

As a further hint that might be of importance: short range correlations are slightly ferromagnetic. In fact this Spin-Liquid phase appears in the vicinity of a ferromagnetic instability, as can be seen both in the case of the multiple-spin exchange Hamiltonian on the triangular lattice, but also for the $J_{1}-J_{2}$ model on the honeycomb lattice [6].

Experimentally, this phase might be observed in the low density $2 \mathrm{~d}$ layers of solid ${ }^{3} \mathrm{He}$ where there is evidence that the coupling constants might be in the good range (see 19,27, 29 and references therein) and possibly in the Wigner crystal 30.

\section{KAGOMÉ-LIKE SPIN-LIQUIDS}

The last situation that we will now discuss is by some details more puzzling. It has been obtained by depleting the triangular lattice, removing one spin out of four: the lattice which is obtained is called the kagomé lattice. Some possible physical realizations are $\mathrm{SrCrGaO}$ or various jarosites 31 33]. The model has been extensively studied in the classical limit [34 38 and it displays an extensive local degeneracy (i.e. an extensive number of modes of zero energy). Some authors have pleaded in favor of a selection of a particular ground-state by the "order out disorder" mechanism [39] but the finite size scaling of spin- $1 / 2$ spectra contradicts this assumption 13]. The system is certainly a Spin-Liquid in the following sense:

- all the correlations (spin-spin, dimer-dimer, chiralchiral, ...) are very small and decay with distance 40.41 .

- the system has a gap for magnetic excitations. Even if it is not sure that the largest size $(\mathrm{N}=36)$ is much larger than all correlation lengths 3 , the finite size scaling studies point to a small spin-gap: probably of the order of one tenth of the coupling constant and quite likely larger than one twentieth of the coupling constant 13,42 .

- the spin-gap is filled by a continuum of singlets and the number of these singlets increases exponentially with the system size $N$ [13,42] as shown in Fig.4.

The understanding of the low lying continuum of singlets is still incomplete but important progresses have been done recently 43 45. Mambrini and Mila 443] have shown that the low lying singlet levels could be related to a family of short range dimer coverings of the lattice. It must be emphasized that short range dimer coverings on this lattice is not necessarily synonymous with exponential decreasing correlations and gapful excitations: the recent results of Mambrini and Mila prove that this is indeed not the case and the comparison between these results and those of Zeng and Elser [45] point to the fact that the non-orthogonality of the dimer coverings plays a major role in this respect. This is an important step forward and we would like now a simple picture of the first excitations.

Ten years ago, Rokhsar and Kivelson have studied a model of hard-core dimers on the square lattive $23,46,48]$. The phase diagram of their model displays two VBC phases separated by a critical point. At

\footnotetext{
${ }^{3}$ The spin-gap of the $N=21,27,36$ samples seems to be in a cross-over regime of scaling, but the energy per spin still evolves as $N^{-3 / 2}$ !
} 
the transition point between the two phases the spectrum of excitations becomes gapless. The low lying modes are the Goldstone modes associated to a quasi LRO in the orientation of the dimers. Let us assume that this hypothesis is qualitatively valid on the kagomé lattice and derive its consequences for the number of states below the spin-gap. Assuming that these Goldstone modes are non-interacting, it is easy to extract from their dispersion law $\left(\epsilon(k)=k^{n}\right)$, the internal energy and entropy versus temperature and thus the number of states below the first triplet excitation. The logarithm of this number of states scales as $N^{\frac{n}{n+2}}$. In Fig.4 we compare the numerical results with this predicted law (assuming $n=1$ or 2). Fixing the unknown constants from the measured value for $N=12$, we see that the prescription is an order of magnitude off at $N=36$ : the $n=1$ mode description gives $\sim 20$ singlet levels in the spin-gap, whereas their real number is 210 t! With the present pieces of information we must conclude that the description of the low lying singlets as Goldstone modes associated to a quasi LRO in the dimer covering is not supported by the numerics 5 . A simple picture of these first excitations is still lacking!

On the other hand, the exponential increase in the number of eigenlevels in a finite band of energy implies a finite entropy per spin at $\mathrm{T}=0$. The same phenomena appearing in each spin sector might be at the origin of the anomalous glassy behavior "without chemical disorder" seen in various compounds (see 49] and refs therein).

Less speculative, the presence of a finite entropy in the singlet channel at low temperature is consistent with the observation of Ramirez and coworkers [50] that the low temperature specific heat of $\mathrm{SrCrGaO}$ is essentially insensitive to large magnetic fields (much larger than the temperature) [51]. This picture is also compatible with the muons experiments of Uemura et al [52 and the elastic spin diffusion measurement of Lee et al [53].

To conclude we must add that these systems seem able to support unconfined spinons excitations with non zero Chern numbers 42.

Up to very recently, we were thinking that this second kind of Spin-Liquid was associated to special lattices as the kagomé or pyrochlore lattices, which at the classical level display an infinite local degeneracy. Some recent re-

\footnotetext{
${ }^{4}$ This number is slightly larger than the one quoted in 42 (some degeneracies were incorrectly numbered).

${ }^{5} \mathrm{~A}$ second check has been done on the density of states versus $\mathrm{E}$ for $N=36$. Due to the intrinsic quantum noise of the numerical density of states, it is impossible with this last criterium to really discriminate between the different hypotheses, but the density of states implied by a mode description $\exp \left(a E^{\frac{2}{n+2}}\right)$ seems less probable than a pure exponential or power law ( the $\chi^{2}$ criterium is approximately twice as large).
}

sults bring new pieces of information: we have observed kagomé-like spectra on the triangular lattice when the 3-sublattice Néel order is destabilized by a frustrating 4-spin exchange [14 and we have no evidence of an infinite local degeneracy at this point. We now conjecture that the interesting feature common to these two cases is the appearance of the "kagomé-like" phase after destabilization of a non-collinear long-range order (either due to quantum fluctuations -when going from the triangular to the kagomé lattice by weakening of bonds 13]-, or to competing frustrating interactions in the multiple-spin exchange problem [14). Contrarily, destabilization of a collinear order (like in section II) leads to dimer LRO.

Is this "kagomé-like behavior" representative of a true new phase or of a quantum critical point? This remains an open question. In the two studied cases we found these characteristics in a rather wide range of parameters: It is nevertheless impossible to exclude a finite size effect. If criticality is to be viewed as a situation where there is no intrinsic length scale nor energy scale and a slowing down of all dynamics, it remains unclear if the proliferation of low lying singlets in this model could not be a signature of criticality (and the above assumption on the scaling of the density of states much too naive!).

\section{MISCELLANEOUS REMARKS AND CONCLUSION}

As a last remark we would like to discuss some questions around the words and concepts of order and disorder. The established expression of "order by disorder" (or "order out of disorder") expresses the fact that quantum fluctuations (as well as thermal fluctuations for which it was primarily introduced by Villain) stabilize the simpler and the more symmetric ordered state amongst the allowed $T=0$ classical ground-states. But more generally the effect of long wave-length quantum fluctuations on a (semi-)classical ground-state might lead to still simpler and more symmetric situations which do not break $S U(2)$ (section III) and even any space symmetries (sections IV and V). In such an issue the word "quantum disorder" has been used (by opposition to Néel long range order) but indeed it should be used with great caution: the ground-state of the Spin-Liquid of Section IV is highly symmetric and very far from a disordered system. Only the "kagomé-like" state might be qualified as "quantum disordered" (section V). To measure this delicate issue one may look at the $T=0$ entropy per spin of the groundstate. In the thermodynamic limit, this quantity scales as $\mathcal{O}(p \operatorname{Ln}(N) / N)$ for a Néel ordered state with $p$ sublattices, as $\mathcal{O}(1 / N)$ in the Valence Bond Crystals (Sec. III) and in the fully gapped Spin-Liquid (Sec. IV), and it is $\mathcal{O}(1)$ in the "kagomé-like" Spin-Liquids.

In conclusion exact diagonalizations have allowed a characterization of 4 different and (probably ) generic 
ground-states of quantum antiferromagnets in $2 \mathrm{~d}$. The two kinds of Spin-Liquids conjectured by P.W. Anderson in 1973 54 have been found (the second with the continuum of singlets seems nevertheless rather different from the initial conjecture). Possible physical realizations of these Spin-Liquids exist. There remain many questions on the first excitations of these Spin-Liquids. Some progress on this subject can probably be done in computing dynamical structure functions with exact diagonalizations schemes, but it would also be useful to devise new analytical approximate tools to look at these questions: exact results on the ground-state and low lying levels of the spectra may hopefully help in achieving this goal!

[1] E. Manousakis, Rev. Mod. Phys. 63, 1 (1991).

[2] H. Schultz and T. Ziman, Europhys. Lett. 8, 355 (1992).

[3] B. Bernu, C. Lhuillier, and L. Pierre, Phys. Rev. Lett. 69, 2590 (1992).

[4] L. Capriotti, A. Trumper, and S. Sorella, Phys. Rev. Lett. 82, 3899 (1999).

[5] J. D. Reger, J. A. Riera, and A. P. Young, J. Phys. Cond. Matt. 1, 1855 (1989).

[6] J.-B. Fouet, P. Sindzingre, and C. Lhuillier, in preparation (unpublished).

[7] H. Neuberger and T. Ziman, Phys. Rev. B 39, 2608 (1989).

[8] D. Fisher, Phys. Rev. B 39, 11783 (1989).

[9] B. Bernu, P. Lecheminant, C. Lhuillier, and L. Pierre, Phys. Rev. B 50, 10048 (1994).

[10] P. Lecheminant, B. Bernu, C. Lhuillier, and L. Pierre, Phys. Rev. B 52, 9162 (1995).

[11] P. Azaria, B. Delamotte, and D. Mouhanna, Phys. Rev. Lett. 70, 2483 (1993).

[12] P. Lecheminant, B. Bernu, C. Lhuillier, and L. Pierre, Phys. Rev. B 52, 6647 (1995).

[13] P. Lecheminant et al., Phys. Rev. B 56, 2521 (1997).

[14] W. LiMing, G. Misguich, P. Sindzingre, and C. Lhuillier, Phys. Rev. B 62, 6372 (2000).

[15] I. Affleck, T. Kennedy, E. Lieb, and H. Tasaki, Phys. Rev. Lett. 59, 799 (1987).

[16] I. Affleck, T. Kennedy, E. H. Lieb, and H. Tasaki, Commun. Math. Phys. 115, 477 (1988).

[17] V. N. Kotov, J. Oitmaa, O. Sushkov, and Z. Weihong, Phys. Rev. B 60, 14613,14616 (1999); L. Capriotti and S. Sorella, Phys. Rev. Lett. 84, 3173 (2000); O. P. Sushkov, J. Oitmaa and Z. Weihong, condmat/0007329; M. S. L. du Croo de Jongh, J. M. J. van Leeuwen and W. van Saarloos, condmat/0002116. This discussion was opened by the large $N$ study: N. Read and S. Sachdev, Phys. Rev. Lett. 66, 1773 (1991); S. Sachdev and N. Read, Phys. Rev. Lett. 77, 4800 (1996).

[18] G. Misguich, B. Bernu, C. Lhuillier, and C. Waldtmann, Phys. Rev. Lett. 81, 1098 (1998).
[19] G. Misguich, C. Lhuillier, B. Bernu, and C. Waldtmann, Phys. Rev. B 60, 1064 (1999).

[20] S. E. Korshunov, Phys. Rev. B 47, 6165 (1993).

[21] K. Kubo and T. Momoi, Z. Phys. B. Condensed Matter 103, 485,489 (1997).

[22] T. Momoi, H. Sakamoto, and K. Kubo, Phys. Rev. B 59, 9491 (1999).

[23] D. Rokhsar and S. Kivelson, Phys. Rev. Lett. 61, 2376 (1988).

[24] N. Read and B. Chakraborty, Phys. Rev. B 40, 7133 (1989).

[25] N. Read and S. Sachdev, Phys. Rev. B 42, 4568 (1990); N. Read and S. Sachdev, Phys. Rev. Lett. 66, 1773 (1991).

[26] M. Oshikawa, Phys. Rev. Lett. 84, 1535 (2000).

[27] M. Roger et al., Phys. Rev. Lett. 80, 1308 (1998).

[28] C. Bauerle et al., Physica B 280, 95 (2000).

[29] K. Ishida, M. Morishita, K. Yawata, and H. Fukuyama, Phys. Rev. Lett. 79, 3451 (1997).

[30] B. Bernu and D. Ceperley, private com (unpublished).

[31] X. Obradors et al., Solid State Commun. 65, 189 (1988).

[32] A. S. Wills et al., Europhys. Lett 42, 325 (1998).

[33] A. P. Ramirez, Annu. Rev. Mater. Sci 24, 453 (1994), and refrences therein.

[34] P. Chandra and P. Coleman, Phys. Rev. Lett. 66, 100 (1991).

[35] I. Richtey, P. Chandra, and P. Coleman, Phys. Rev. B 47, 15342 (1993).

[36] A. Harris, C. Kallin, and A. Berlinsky, Phys. Rev. B 45, 2899 (1992).

[37] J. Chalker, P. Holdsworth, and E. Shender, Phys. Rev. Lett. 68, 855 (1992).

[38] E. Shender, V. Cherepanov, P. Holdsworth, and A. Berlinsky, Phys. Rev. Lett. 70, 3812 (1993).

[39] A. Chubukov, Phys. Rev. Lett. 69, 832 (1992).

[40] J. Chalker and J. Eastmond, Phys. Rev. B 46, 14201 (1992).

[41] P. Leung and V. Elser, Phys. Rev. B 47, 5459 (1993).

[42] C. Waldtmann et al., Eur. Phys. J. B 2, 501 (1998).

[43] M. Mambrini and F. Mila, cond-mat/0003080 (unpublished).

[44] F. Mila, Phys. Rev. Lett. 81, 2356 (1998).

[45] C. Zeng and V. Elser, Phys. Rev. B 42, 8436 (1990).

[46] P. Leung, K. Chiu, and K. Runge, Phys. Rev. B 54, 12938 (1996).

[47] E. Fradkin, in Field Theories of Condensed Matter Systems, Frontiers in Physics, edited by D. Pines (AddisonWesley, USA, 1991).

[48] S. Sachdev and M. Vojta, J. Phys. Soc. Jpn. Supp.B, 1 (2000).

[49] A. Wills et al., cond-mat/0001344; to appear in Phys. Rev. B., Rap. Com. 62,(2000).

[50] A. P. Ramirez, B. Hessen, and M. Winkelmann, Phys. Rev. Lett. 84, 2957 (2000).

[51] P. Sindzingre et al., Phys. Rev. Lett. 84, 2953 (2000).

[52] Y. Uemura et al., Phys. Rev. Lett. 73, 3306 (1994).

[53] S.-H. Lee et al., Europhys. Lett 35, 127 (1996).

[54] P. Anderson, Mater. Res. Bull. 8, 153 (1973). 


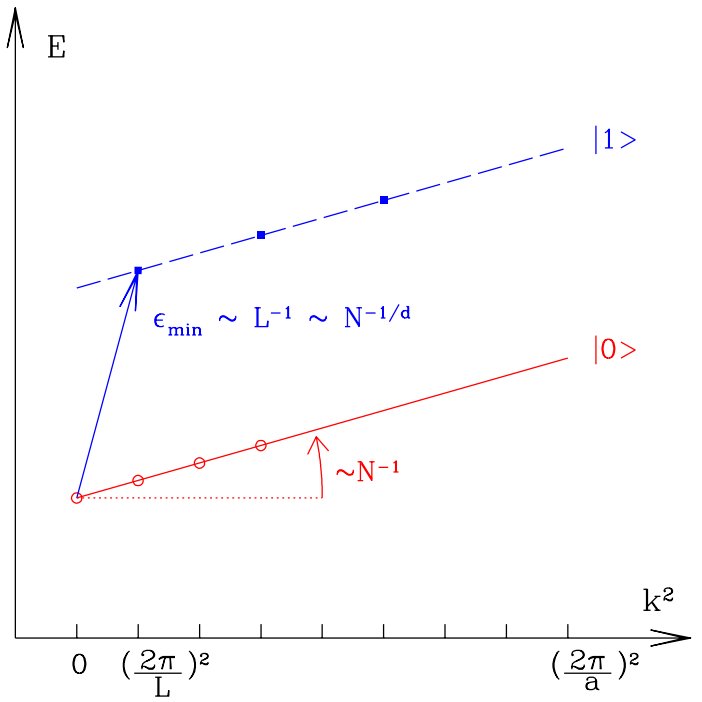

FIG. 1. Typical spectrum of a finite size solid. The first tower of eigenlevels $|0\rangle$ is associated with c.o.m. motion. The second tower $|1\rangle$ describes the softest phonon.

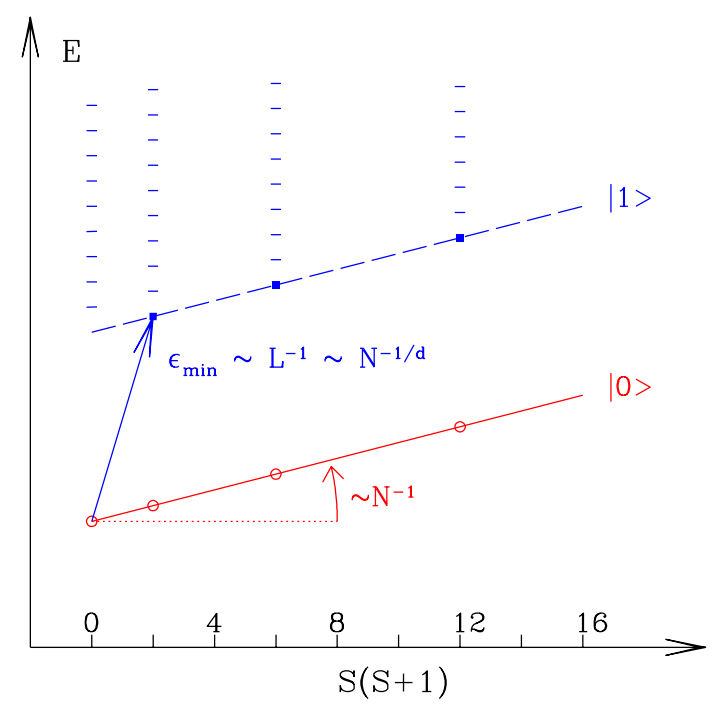

FIG. 2. Typical spectrum of a finite size collinear antiferromagnet with a Néel order. The tower of eigenlevels $|0\rangle$ are the QDJS associated with the free dynamics of the order parameter. The second tower $|1\rangle$ is associated with the lowest magnon.

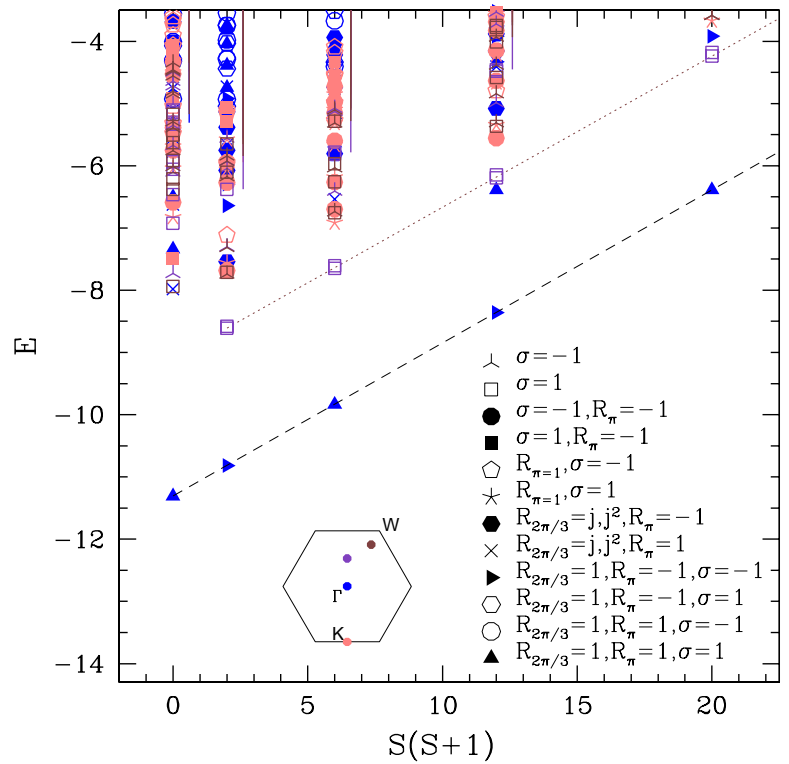

FIG. 3. Antiferromagnetic Heisenberg model on the honeycomb lattice: eigenenergies vs the eigenvalues of $\mathbf{S}^{2}$. The dashed-line is a guide to the eyes for the QDJS. The dotted line joins the states associated to the first magnon. There is one QDJS for each $S$ (as expected for a collinear antiferromagnet): they are $\mathbf{k}=\mathbf{0}$ states, invariant under a $2 \pi / 3$ rotation around an hexagon center, even (odd) under inversion, odd (even) under a reflexion with respect to an axis going through nearest neighbor hexagon centers for $S$ even (odd).

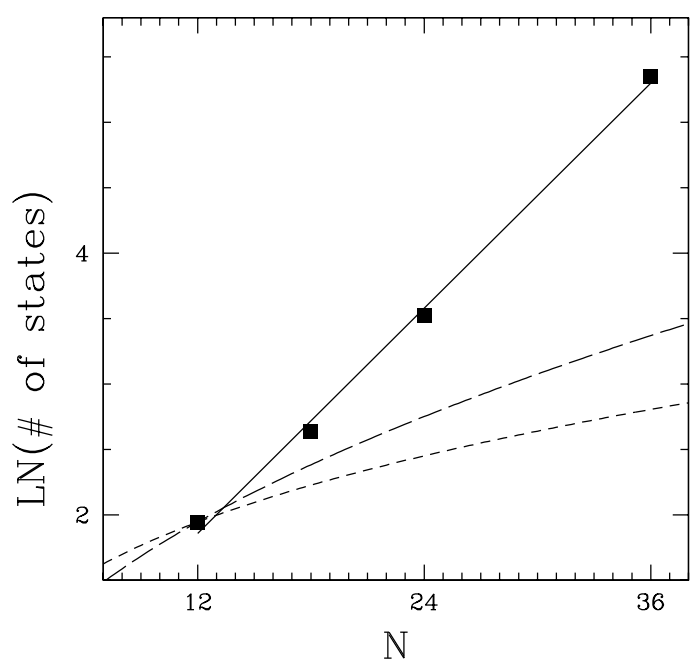

FIG. 4. Logarithm of the number of states in the magnetic gap on the kagomé lattice (squares fitted by a continous line) vs the size $N$ of the system. The observed behavior differs from the scaling law $\exp \left(b N^{n /(n+2)}\right)$ deduced from a single mode description of the continuum with a dispersion law $\epsilon(k)=k^{n}$ for $n=1$ (short-dashed line) and $n=2$ (long-dashed line). 